\title{
The effects of compound centella formula on OxInflammation and silent information regulator 1 in a high-fat diet/streptozotocin-induced diabetic kidney disease rat model
}

\author{
QIN ZHU ${ }^{1}$, XIAO-HONG LI ${ }^{1}$, HONG-YU CHEN ${ }^{1}$ and QIN-YANG JIN ${ }^{2}$ \\ ${ }^{1}$ Department of Nephrology, Key Laboratory of Zhejiang Province, Management of Kidney Disease, \\ Hangzhou Hospital of Traditional Chinese Medicine, Hangzhou, Zhejiang 310007; \\ ${ }^{2}$ Department of Cardiology, Zhejiang Provincial People's Hospital, People's Hospital of \\ Hangzhou Medical College, Hangzhou, Zhejiang 310014, P.R. China
}

Received March 7, 2020; Accepted April 14, 2021

DOI: $10.3892 /$ etm.2021.10394

\begin{abstract}
The Chinese decoction compound centella formula (CCF) is clinically effective against diabetic kidney disease (DKD), but the exact mechanism remains unclear. The present study aimed to investigate the effects of CCF on OxInflammation and silent information regulator 1 (SIRT1) levels in rats with streptozotocin (STZ)-induced diabetes. Sprague-Dawley rats were divided into CCF, losartan, diabetic control (DC) and normal control (NC) groups $(n=7)$. Except for the NC, all subgroups of rats were fed a high-fat diet for 112 days and received a single intraperitoneal injection of
\end{abstract}

Correspondence to: Dr Qin-Yang Jin, Department of Cardiology, Zhejiang Provincial People's Hospital, People's Hospital of Hangzhou Medical College, 158 Shangtang Road, Hangzhou, Zhejiang 310014, P.R. China

Email: jqy119@163.com

Dr Hong-Yu Chen, Department of Nephrology, Key Laboratory of Zhejiang Province, Management of Kidney Disease, Hangzhou Hospital of Traditional Chinese Medicine, 453 Stadium Road, Hangzhou, Zhejiang 310007, P.R. China

E-mail: hzchenhy@126.com

Abbreviations: CCF, compound centella formula; DKD, diabetic kidney disease; SIRT1, silent information regulator 1; STZ, streptozotocin; LST, losartan; DC, diabetic control; NC, normal control; HPLC, high-performance liquid chromatography; FBG, fasting blood glucose; UPCR, urine protein-to-creatinine ratio; Scr, serum creatinine; BUN, blood urea nitrogen; PAS, Periodic acid Schiff; HE, haematoxylin-eosin; SOD, superoxide dismutase; MDA, malondialdehyde; NOX4, nicotinamide adenine dinucleotide phosphate oxidase 4; TNF- $\alpha$, tumour necrosis factor- $\alpha$; NF- $\kappa \mathrm{B}$, nuclear factor- $\kappa \mathrm{B}$; ROS, reactive oxygen species; IOD, integral optical density; AOD, average optical density; RT-qPCR, reverse transcription-quantitative PCR

Key words: compound centella formula, diabetic kidney disease, OxInflammation, silent information regulator 1
$35 \mathrm{mg} / \mathrm{kg} \mathrm{STZ}$ on day 29. All rats were sacrificed on day 112 . High-performance liquid chromatography was performed to analyse asiaticoside, astragaloside and triptolide levels in CCF $(0.3400,0.0640$ and $0.0001 \mathrm{mg} / \mathrm{ml}$, respectively). Fasting blood glucose, urine protein-to-creatinine ratio, serum creatinine and blood urea nitrogen were quantified. Periodic acid Schiff staining, H\&E staining and transmission electron microscopy were used to examine kidney pathological changes. The mRNA and protein expression levels of SIRT1 in renal tissues were analysed by reverse transcription-quantitative PCR, western blotting and immunohistochemistry. Oxidative stress was evaluated by measuring the levels of superoxide dismutase (SOD), malondialdehyde (MDA) and nicotinamide adenine dinucleotide phosphate oxidase 4 (NOX4) in renal tissues. TNF- $\alpha$ and NF- $\kappa$ B p65 subunit in renal tissues were assessed for inflammation. Compared with the rats in the NC group, the rats in the DC group exhibited renal injury with proteinuria, decreased expression levels of SIRT1 and SOD $(\mathrm{P}<0.01)$ and increased levels of MDA, NOX4, TNF- $\alpha$ and NF- $\kappa \mathrm{B}$ p65 $(\mathrm{P}<0.01)$. CCF treatment reduced proteinuria $(\mathrm{P}<0.01)$, alleviated renal damage, decreased MDA, NOX4, TNF- $\alpha$ and NF- $\kappa$ B p65 levels $(\mathrm{P}<0.01)$, increased SOD levels $(\mathrm{P}<0.05)$ and increased SIRT1 mRNA and protein expression levels $(\mathrm{P}<0.01)$. The present study indicates that $\mathrm{CCF}$ effectively protects the kidney from diabetes by inhibiting OxInflammation and upregulating SIRT1.

\section{Introduction}

Diabetes mellitus, also known simply as diabetes, is one of the most serious health-threatening diseases worldwide. According to the Austrian Diabetes Report, in 2017, 415 million people worldwide lived with diabetes (1). Diabetic kidney disease (DKD) is one of the major microvascular complications of diabetes and is the most common cause of end-stage renal disease worldwide (2). Therefore, it is urgent to explore new prevention and treatment strategies for DKD. The pathogenesis of DKD has not been fully elucidated. The complex pathogenesis of DKD mainly focuses on hyperglycaemia, lipid metabolism disorders, abnormal haemodynamics, inflammatory cytokines, 
oxidative stress and cell apoptosis $(3,4)$. An increasing body of research has confirmed that oxidative stress and inflammation play crucial roles in DKD $(5,6)$. Inflammation can lead to excessive reactive oxygen species (ROS), which can cause lipid peroxidation reactions and decreased cellular antioxidant capacity (7). In contrast, ROS and ROS-induced DNA damage can promote inflammatory responses and fibrotic processes (8). The term 'OxInflammation' refers to a prepathological condition; it has been well-documented that mild-subclinical chronic inflammation is related to chronic and systemic oxidative stress with in a vicious circle (9). Silent information regulator 1 (SIRT1) is a nicotinamide adenosine dinucleotide-dependent protein deacetylase with remarkable abilities to prevent diseases and even reverse aspects of ageing. It can regulate a variety of biological processes, including inflammatory and metabolic disorders $(10,11)$. Renoprotective effects of SIRT1 have been found in experimental models of renal disorders, including DKD $(12,13)$.

Although many interventions have been found to be effective against DKD, including lifestyle adjustment, glycaemic control, blood pressure control, renin-angiotensin system blockers, sodium-dependent glucose transporter-2 inhibitors and glucagon-like peptide-1 agonists, few have been established as optimal options (2). The discovery of new interventions, which overcome these limitations (such as restricted use in chronic renal failure and non-specific effect of reducing albuminuria) to delay the evelopment of DKD is warranted. At present, the use of medicinal plants and their natural components as future drugs for the treatment of diabetes and its complications has received considerable interest from researchers worldwide. The Chinese decoction compound centella formula (CCF) was designed by a famous contemporary doctor of traditional Chinese medicine, Professor Yongjun Wang (14). The composition and daily dose for adults of this decoction are as follows: Centella asiatica (L.) Urb. (JiXueCao), $30 \mathrm{~g}$; Astragalus Membranaceus Fish. (HuangQi), $30 \mathrm{~g}$; Tripterygium wilfordii Hook. f. (LeiGongTeng), $15 \mathrm{~g}$. In a previous study, it was observed that $\mathrm{CCF}$ reduced the quantity of proteins found in the $24 \mathrm{~h}$ urinary protein test, improved renal function, inhibited mesangial cell proliferation and mesangial matrix accumulation, and prevented sclerosis of the glomerulus in stage 3-4 patients with DKD treated for 3 months. A small number of patients $(2 / 43)$ had a drug-related increase in alanine transaminase, which was within 3 times the normal value and returned to normal levels after symptomatic treatment (15). Although CCF has been indicated to be an effective treatment for DKD in a clinical setting, the exact mechanism is still unclear.

In the present study, the protective effects of CCF against renal injuries in streptozotocin (STZ)-induced diabetic rats were examined, along with the possible mechanisms associated with the inhibition of crosstalk between inflammatory responses and oxidant stress and the regulation of SIRT1.

\section{Materials and methods}

Animals and experimental design. A total of 30 male Sprague Dawley rats (specific-pathogen-free grade; 4-5 weeks old, weighing $100 \pm 10 \mathrm{~g}$ ) were purchased from Shanghai SIPPR-Bk Laboratory Animal Co., Ltd. The experiment was approved by the Zhejiang Chinese Medical University Animal Ethics Committee. The rats were reared in a standard experimental animal laboratory with free access to food and water in a specific pathogen-free laboratory environment under the following conditions: Temperature, $20-25^{\circ} \mathrm{C}$; humidity, 50-65\% and a $12 \mathrm{~h}$ light/dark cycle. A total of 7 rats were fed ordinary feed to represent the normal control (NC). Considering the mortality rate of the pathological models, 23 rats were fed high-fat feed for 112 days $(72.5 \%$ ordinary feed formula plus $10 \%$ lard, $10 \%$ sucrose, $2.0 \%$ cholesterol, $0.5 \%$ cholic acid and $5 \%$ yolk powder). The feed was purchased from Beijing Boaigang Biotechnology Co., Ltd. On the 29th day of the experiment, the same 23 rats all received a single intraperitoneal injection of STZ (MilliporeSigma Canada Co.) at a dose of $35 \mathrm{mg} / \mathrm{kg}$; two of the 23 rats gradually lost weight after injection of STZ and appeared to show slower activity, as well as eating less food and grooming less. For ethical reasons, these two rats were eventually sacrificed. It was speculated that the abnormal response of the two rats was due to a faulty intraperitoneal injection procedure. The remaining 21 rats were randomly divided into the diabetic control (DC) group (n=7), CCF group $(n=7)$ and losartan (LST) group $(n=7)$. As a classic drug for the treatment of diabetic nephropathy, LST was used as the positive control drug for centella formula in this experiment. Plasma fasting blood glucose (FBG) was measured from the tail vein, and FBG levels $>16.7 \mathrm{mmol} / 1$ ( $72 \mathrm{~h}$ after injection) were considered diabetic rats (14). Subsequently, the drug intervention was carried out from the 32 nd to the 112 th day. The rats in the CCF group were treated with a concentrated solution of CCF at $2 \mathrm{ml} /$ day per rat. The dose of losartan potassium was $4.5 \mathrm{mg} / \mathrm{kg} /$ day per rat (diluted to $2 \mathrm{ml}$ with normal saline) in the LST group (Merck Sharp \& Dohme-Hoddesdon). The rats in the NC and DC groups received the same volume of normal saline. The drug intervention was administered by gavage. All rats were sacrificed by decapitation on the 112th day of the experiment after anaesthesia. Firstly, the midline of the abdomens of rats were cut open following local skin disinfection. The kidneys were then carefully dissociated, and the kidney blood vessels were clamped with hemostatic forceps and ligated. The kidneys were then removed, and the abdomens were sutured.

Preparation of $C C F$. One daily dose of CCF for each rat included $0.8 \mathrm{~g}$ of Centella asiatica (L.) Urb. (JiXueCao), $0.8 \mathrm{~g}$ of Astragalus membranaceus Fish. (HuangQi), and $0.4 \mathrm{~g}$ of Tripterygium wilfordii Hook. f. (LeiGongTeng). The rat $\mathrm{CCF}$ dose was calculated based on the human dose according to the body surface area formula (16): $\mathrm{A}=\mathrm{k}\left(\mathrm{W}^{2 / 3}\right) / 10,000$, where $\mathrm{k}=9.1$ (A, Body surface area, $\mathrm{m}^{2}$; K, A constant, which varies with animal species; $\mathrm{W}$, weight, $\mathrm{g}$ ). The herbs were mixed in water, decocted for $45 \mathrm{~min}$ and then concentrated. The final volume of the concentrated decoction was $2 \mathrm{ml}$ for one rat per day. The decoction was stored at $4^{\circ} \mathrm{C}$.

Chemical analysis of CCF extracts by high-performance liquid chromatography. To prepare the asiaticoside reference solution, $6.75 \mathrm{mg}$ asiaticoside reference (National Institute for the Control of Pharmaceutical and Biological Products) was precisely weighed and added to methanol to obtain a $0.27 \mathrm{mg} / \mathrm{ml}$ solution. To prepare the sample solution, $5 \mathrm{ml}$ of 
this solution was added to a test tube and heated $\left(100^{\circ} \mathrm{C}\right)$ in a water bath for evaporation until dry. Subsequently, the solution was dissolved in methanol and placed in an ultrasonic bath for $30 \mathrm{~min}$. The final volume was $2 \mathrm{ml}$ in a volumetric flask. After shaking well, the extraction solution was filtered with a $0.45 \mu \mathrm{m}$ filter membrane. For content analysis, the precise absorption of the reference asiaticoside solution $(10 \mu \mathrm{l})$ and sample solution $(10 \mu \mathrm{l})$ was analysed by injecting the samples into a liquid chromatograph (Varian ProStar 230 Solvent delivery module; Varian) with the following conditions: Column, YMC ODS C18 (YMC Co., Ltd.) inner diameter: $4.6 \mathrm{~mm}$, length: $250 \mathrm{~mm}$; particle diameter: $5 \mu \mathrm{m}$ ); mobile phase, acetonitrile:water (30:70); atomization temperature, $40^{\circ} \mathrm{C}$; gasification temperature, $90^{\circ} \mathrm{C}$; flow rate, $1.0 \mathrm{ml} / \mathrm{min}$; nitrogen flow rate, $1.6 \mathrm{l} / \mathrm{min}$.

To prepare the astragaloside reference solution, $7.50 \mathrm{mg}$ astragaloside reference (National Institute for the Control of Pharmaceutical and Biological Products) was precisely weighed, and methanol was added $(0.30 \mathrm{mg} / \mathrm{ml})$. To prepare the sample solution, $30 \mathrm{ml}$ sample solution was extracted by adding $40 \mathrm{ml}$ of $80 \%$ methanol as the solvent overnight; $20 \mathrm{ml}$ methanol solvent was added again. Subsequently, the resultant mixture was heated under reflux for $4 \mathrm{~h}$. The methanol extract was evaporated to dryness, and the desiccated residue was dissolved in water. The solution was then extracted four times with $40 \mathrm{ml}$ of n-butanol and washed in $40 \mathrm{ml}$ ammonia solution twice. After discarding the ammonia solution, n-butanol was evaporated to dryness, and the residue was dissolved in $5 \mathrm{ml}$ of water. Then, the crude extracts were filtered through a D101 macroporous adsorption resin column (length, $12 \mathrm{~cm}$; inner diameter. $1.5 \mathrm{~cm}$ ). A total of $50 \mathrm{ml}$ of water was used as the eluent and discarded later, followed by ethanol elution. The solution was eluted with $40 \%$ ethanol (30 $\mathrm{ml})$, and the eluate was discarded. The solution was then eluted again with $70 \%$ ethanol $(80 \mathrm{ml})$. The eluent solution was collected and evaporated to dryness. The residue was dissolved in methanol and transferred to a $5 \mathrm{ml}$ volumetric flask. After the addition of methanol to the flask (to $5 \mathrm{ml}$ ), the sample was shaken. For content analysis, the precision absorption of the astragaloside reference solution (10 $\mu \mathrm{l})$ and sample solution $(10 \mu \mathrm{l})$ was measured by liquid chromatography (Varian ProStar 230 Solvent delivery module; Varian) with the following conditions: Column, YMC ODS C18 (YMC Co., Ltd.)(inner diameter: $4.6 \mathrm{~mm}$, length: $250 \mathrm{~mm}$; particle diameter: $5 \mu \mathrm{m})$; mobile phase, acetonitrile:water (35:65); the other details were the same as those aforementioned.

To prepare the triptolide reference solution, $3.01 \mathrm{mg}$ triptolide reference (content $\geq 98 \%$; National Institute for the Control of Pharmaceutical and Biological Products) was precisely weighed, and methanol was added $(0.03 \mathrm{mg} / \mathrm{ml})$. To prepare the sample solution, $5 \mathrm{ml}$ of solution was added to a test tube. Water was used to dilute the solution to $25 \mathrm{ml}$. The supernatant was collected after centrifugation $(5,702 \mathrm{x} \mathrm{g}$, room temperature) and heated in a water bath for evaporation until dry. Subsequently, methanol $(2.5 \mathrm{ml})$ and methylene chloride $(2.5 \mathrm{ml})$ were added to the residue in neutral alumina (10 g; diameter, $1.5 \mathrm{~cm}$; wet packing column). A mixture of methanol and methylene chloride (1:3) was used for elution. The eluent was collected at $100 \mathrm{ml}$ and steamed. The residue was dissolved in methanol and transferred to a $5 \mathrm{ml}$ volumetric flask. After shaking well, the extraction solution was filtered with a $0.45 \mu \mathrm{m}$ filter membrane. For content analysis, the precise absorption of the triptolide reference solution $(10 \mu \mathrm{l})$ and sample solution $(10 \mu \mathrm{l})$ were analysed by liquid chromatography (Varian ProStar 230 Solvent delivery module; Varian) with the following conditions: Column, YMC ODS C18 (YMC Co., Ltd.) (inner diameter: $4.6 \mathrm{~mm}$, length: $250 \mathrm{~mm}$; particle diameter: $5 \mu \mathrm{m}$ ); mobile phase, acetonitrile:water (30:70); detection wavelength, $220 \mathrm{~nm}$; the other details were the same as those aforementioned.

Analysis of FBG, urine protein-to-creatinine ratio (UPCR), serum creatinine $(\mathrm{Scr})$ and blood urea nitrogen $(B U N)$. FBG was measured from the tail vein on the 1st, 32nd, 42nd, 56th, 84th and 112th days of the study using Accu-Chek Performa glucometers [Roche Diagnostics (Shanghai) Co., Ltd.]. Metabolic cages were used to collect urine. The UPCR was determined by the pyrogallol red method (MilliporeSigma) using an AU5800 automatic biochemical analyser (Beckman Coulter, Inc.) on the 1st and 112th day. The rats were anaesthetised prior to blood draws ( $\sim \mathrm{ml}$ per rat). All the rats were anaesthetised with intraperitoneal administration of $10 \%$ chloral hydrate (400 mg/kg) (17). Scr and BUN were measured using an AU5800 automatic biochemical analyser (Beckman Coulter, Inc.) on the 112th day.

Renal histology analysis by $H \& E$ staining and periodic acid-Schiff (PAS) staining. One side of the rat kidney was fixed with $10 \%$ neutral buffered formalin (at room temperature $\geq 24 \mathrm{~h}$ ), dehydrated with gradient alcohol (at room temperature, 75\% x2, $30 \mathrm{~min}$; $95 \% \times 3,30 \mathrm{~min}$; and 100\% x2, $30 \mathrm{~min}$ ), cleared with xylene, waxed, embedded and sectioned $(3 \mu \mathrm{m})$. Tissue sections were deparaffinized in xylene and stained with $\mathrm{H} \& \mathrm{E}$ for $20 \mathrm{~min}$ at room temperature. For PAS staining, histological sections were deparaffinized, oxidized in $1 \%$ aqueous periodate solution for $15 \mathrm{~min}$ and washed three times in distilled water. Sections were then soaked in Schiff solution for $15 \mathrm{~min}$. Then, the sections were rinsed under running water for $15 \mathrm{~min}$. Subsequently, the nuclei were stained with hematoxylin (room temperature for $10 \mathrm{~min}$ ) followed by differentiation with ethanol-hydrochloric acid. The areas of nuclei appeared blue, while the basal membrane of the glomerulus and renal tubules, cytoplasm, mesangial matrix of the glomerulus and collagen fibers were red under light microscopy (magnification, $\mathrm{x} 200$ or $\mathrm{x} 400)(\mathrm{BX} 51$; Olympus Corporation). The relative mesangial matrix index and the relative glomerular volume were calculated $(18,19)$.

Ultrastructure of the kidney through transmission electron microscopy. The rat renal cortical tissue samples (1x1x1 mm) were collected and fixed with $2.5 \%$ glutaraldehyde $(60 \mathrm{~min})$ and $1 \%$ osmic $(120 \mathrm{~min})$ acid at $4^{\circ} \mathrm{C}$ After dehydration, the tissues were embedded in epoxy resin. The ultrathin sections $(100 \mathrm{~nm})$ were stained with uranium acetate-lead citrate and then examined with a transmission electron microscope (JEM1400; JEOL, Ltd.). The foot process fusion rate of the podocyte in each specimen was calculated $(20,21)$.

Analysis of SOD and MDA activities in rat renal tissues. SOD activity and MDA content were analysed with SOD test 
Table I. Primer sequences used for the quantitative real-time polymerase chain reaction.

\begin{tabular}{lcl}
\hline Gene & Forward primer $\left(5^{\prime}-3 '\right)$ & Reverse primer $(5 '-3$ ') \\
\hline SIRT1 $(141 \mathrm{bp})$ & GCTCGCCTTGCTGTGGACTTC & GTGACACAGAGATGGCTGGAACTG \\
GAPDH $(252 \mathrm{bp})$ & ACAGCAACAGGGTGGTGGAC & TTTGAGGGTGCAGCGAACTT
\end{tabular}

SIRT1, silent information regulator 1 .

Table II. Concentrations of representative components in CCF.

\begin{tabular}{lc}
\hline Standard substance & Concentration in CCF $(\mathrm{mg} / \mathrm{ml})$ \\
\hline Asiaticoside & 0.3412 \\
Astragaloside & 0.0635 \\
Triptolide & 0.0001 \\
\hline
\end{tabular}

$\mathrm{CCF}$, compound centella formula.

kits (WST-1 method; cat.no. A001-3-1; Nanjing Jiancheng Bioengineering Institute) and MDA test kits (TBA method; cat.no. A003-1-1; Nanjing Jiancheng Bioengineering Institute) according to the manufacturer's instructions.

ELISA assay for NF- $\kappa B$ p65 and TNF- $\alpha$ in kidney tissues. The levels of TNF- $\alpha$ and NF- $\mathrm{NB}$ p 65 in the rat kidney tissues were measured using TNF- $\alpha$ (cat. no. ELK1396; ELK Biotechnology, Ltd.) and NF- $\kappa$ B p 65 ELISA kits (cat. no. ELK5691; ELK Biotechnology, Ltd.) according to the manufacturer's instructions.

Reverse transcription-quantitative PCR (RT-qPCR) for SIRTI expression level. Total RNA from the rat kidney tissues was extracted using the TRIzol ${ }^{\circledR}$ method (Invitrogen; Thermo Fisher Scientific, Inc.). The total RNA concentration was analysed by a UV spectrophotometer $(260 \mathrm{~nm} / 280 \mathrm{~nm})$. Then, cDNA was synthesized using a PrimeScript ${ }^{\mathrm{TM}}$ RT reagent Kit with gDNA Eraser (Takara Bio, Inc.). Subsequently, qPCR was performed using a SYBR Green real-time PCR kit (Takara Bio, Inc.). GAPDH was used as a reference gene. The primer sequences are presented in Table I. The expression levels of mRNA were quantified using the $2^{-\Delta \Delta \mathrm{Cq}}$ method (22).

Western blotting for SIRT1 and NOX4 protein expression level in renal tissue. Protein contents of the rat renal tissues were quantified using the BCA method. 5\% SDS-PAGE gels were prepared. Subsequently, a sample buffer was added to the protein samples $(50 \mu \mathrm{g} /$ lane $)$. The mixtures were denatured at $95^{\circ} \mathrm{C}$ for $10 \mathrm{~min}$ before electrophoresis was performed. After migration, all PVDF membranes were blocked with $5 \%$ skim milk for $30 \mathrm{~min}$ at room temperature, followed by incubation with primary antibodies against SIRT1 (1:600; cat. no. 13161-1-AP; Wuhan Sanying Biotechnology) and NOX4 (1:600; cat. no. 14347-1-AP; Wuhan Sanying Biotechnology) overnight at $4^{\circ} \mathrm{C}$. After TBST washing, the membranes were incubated with secondary antibody (anti-rabbit; 1:10,000; cat. no. DW-GAR007; Jackson ImmunoResearch Laboratories, Inc.) for $60 \mathrm{~min}$ at room temperature. An enhanced chemiluminescence reagent was used for visualization (Applygen Technologies Inc.), and Image-Pro Plus 6.0 software (Media Cybernetics, Inc.) was employed to analyse the results.

Immunohistochemistry assay for SIRT1 protein expression level determination and localisation in kidney tissues. Blocks of rat kidney tissues smaller than $0.5 \times 0.5 \times 0.1 \mathrm{~cm}$ were taken for fixation with $10 \%$ neutral buffered formalin (room temperature for $\geq 24 \mathrm{~h}$ ), dehydrated with an alcohol gradient (room temperature, 75\% x2, $30 \mathrm{~min}$; 95\% x3, $30 \mathrm{~min}$; and 100\% x2, $30 \mathrm{~min}$ ), cleared with xylene, waxed, embedded and sectioned $(3 \mu \mathrm{m})$. Then, the sections were treated with citric acid antigen repair buffer and washed with PBS three times. Sections were blocked using 10\% normal goat serum (cat. no. SL038; Beijing Solarbio Science \& Technology Co., Ltd.) for $20 \mathrm{~min}$ at room temperature. The primary antibody against SIRT1 (1:50; cat. no. 13161-1-AP; Wuhan Sanying Biotechnology) was added, the slides were incubated at $4^{\circ} \mathrm{C}$ overnight and washed with PBS. Secondary antibodies (anti-rabbit; 1:50,000; cat. no. sp-9001; Jackson ImmunoResearch Laboratories, Inc.) were added $\left(37^{\circ} \mathrm{C}\right.$ for $\left.20 \mathrm{~min}\right)$. Then, the sections were washed with PBS three times. Finally, immunostaining was visualized using 3,3'-diaminobenzidine and counterstained with haematoxylin (room temperature for $1 \mathrm{~min}$ ). The results were observed under an inverted microscope (BX43, Olympus Corporation) at a magnification of $\mathrm{x} 400$. Brown staining was considered positive and the staining intensity was calculated as the integral optical density (IOD). Five IODs for each section were randomly collected for semi-quantitative analysis. The average optical density (AOD) was calculated using Image-Pro Plus 6.0.

Statistical methods. Statistics were performed using SPSS 19.0 software (IBM Corp.). All data are presented as the mean \pm standard deviation. The significant differences among the four groups were analysed by one-way ANOVA, followed by post hoc comparison with Tukey's (equal variances assumed) or Dunnett's T3 test (equal variances not assumed). $P<0.05$ was considered to indicate a statistically significant difference.

\section{Results}

Concentration of representative components in CCF. In a previous study (14), the concentration of representative components in CCF was measured. The representative chemical 
A

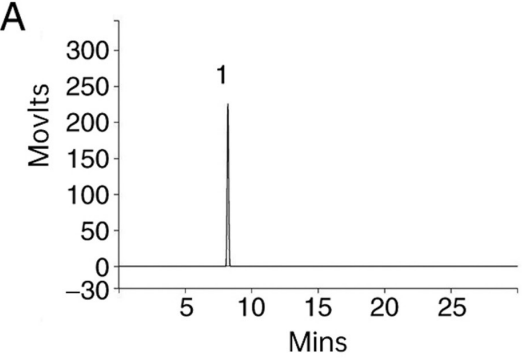

C

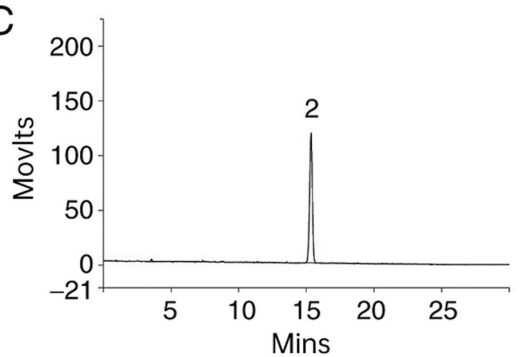

E

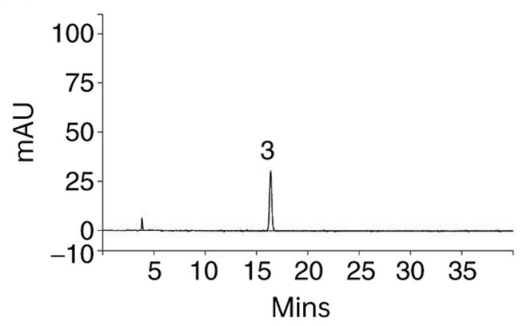

B

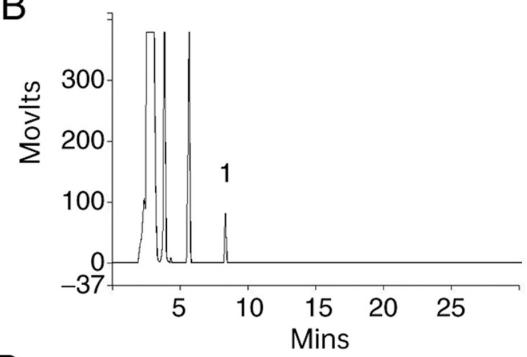

D

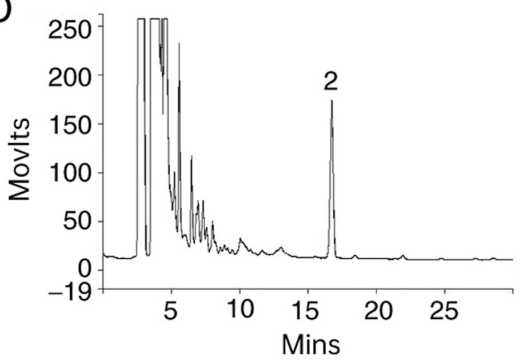

$\mathrm{F}$

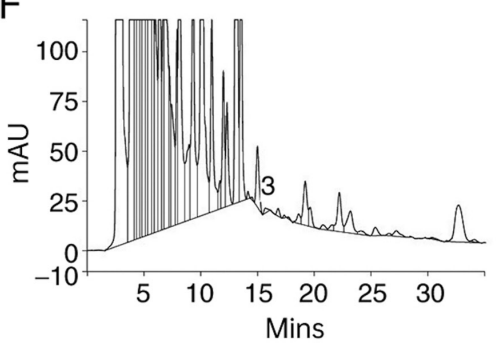

Figure 1. HPLC detection of the asiaticoside (A) standard and (B) asiaticoside in CCF. HPLC detection of the astragaloside (C) standard and (D) astragaloside in CCF. HPLC detection of the triptolide (E) standard and (F) triptolide in CCF. HPLC, high-performance liquid chromatography; CCF, compound centella formula; 1 , asiaticoside; 2 , astragaloside; 3 , triptolide.
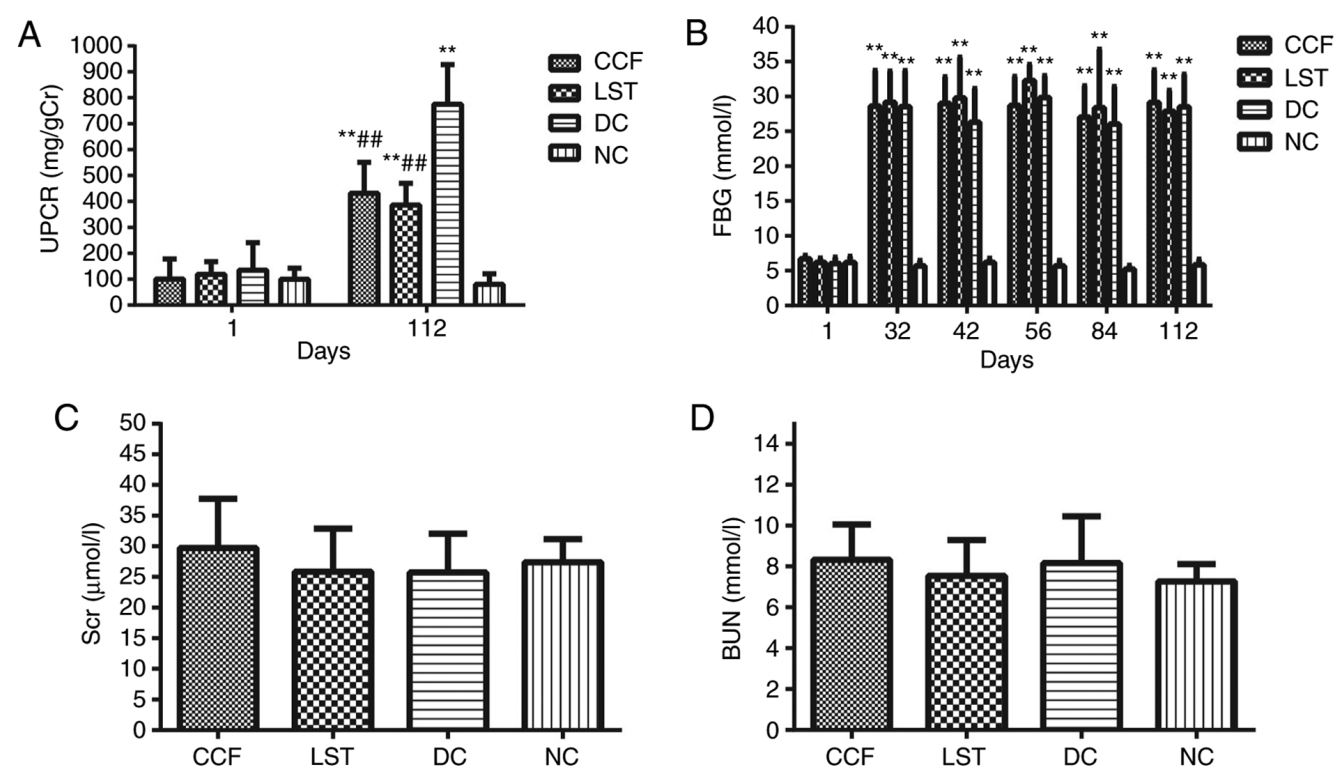

Figure 2. (A) UPCR, (B) FBG, (C) Scr and (D) BUN were measured in four groups of Sprague-Dawley rats (n=7). Data are expressed as the mean \pm standard deviation. ${ }^{* *} \mathrm{P}<0.01$ vs. the NC group, ${ }^{\# \#} \mathrm{P}<0.01$ vs. the $\mathrm{DC}$ group. UPCR, urine protein-to-creatinine ratio; FBG, fasting blood glucose; Scr, serum creatinine; BUN, blood urea nitrogen; CCF, compound centella formula; LST, losartan (4.5 mg/kg/day); DC, diabetic control; NC, normal control.

components in CCF were detected by high-performance liquid chromatography (Table II). The peak retention times and concentrations of asiaticoside, astragaloside and triptolide were measured carefully (Fig. 1)
General conditions. The rats in the DC group exhibited dull fur, weight loss, increased appetite, thirst and frequency of urination compared with the rats in the NC group. In the LST and CCF groups, these symptoms were improved. 
A
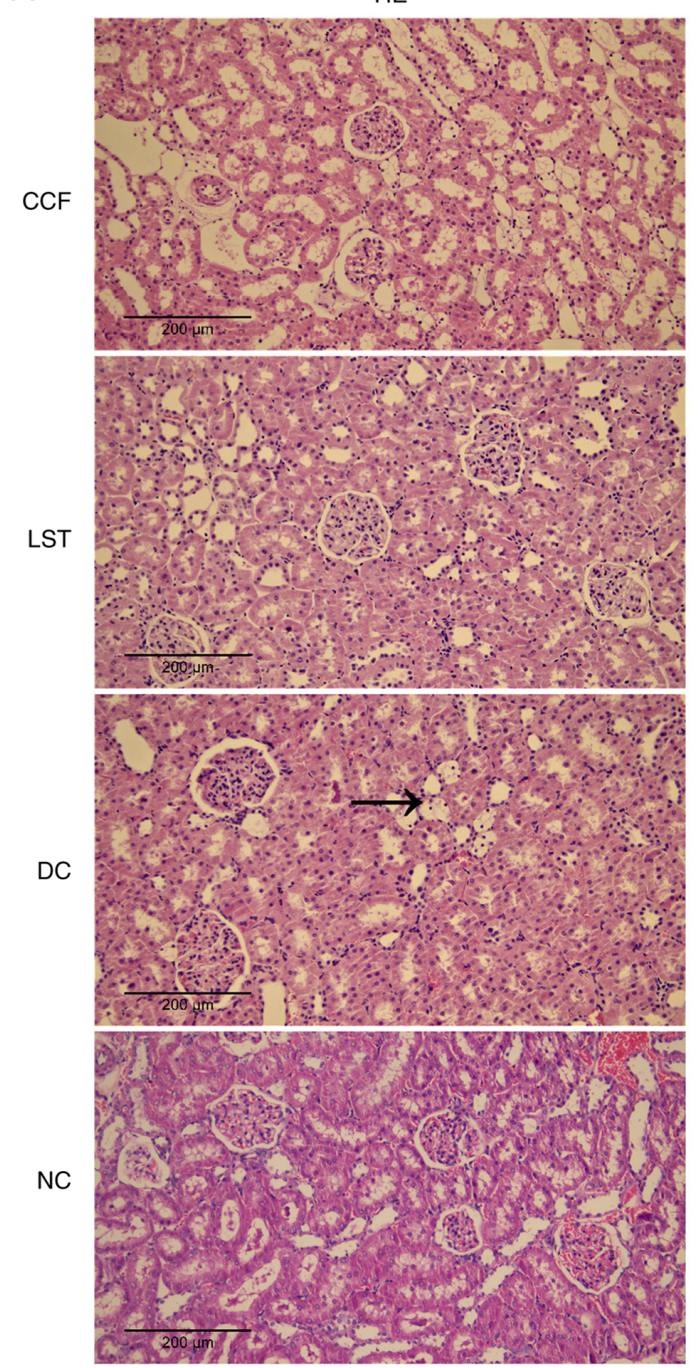

C

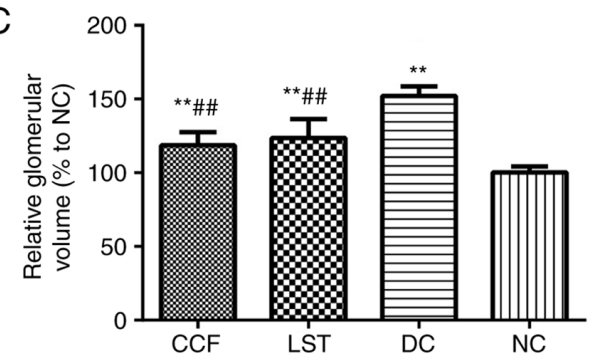

B
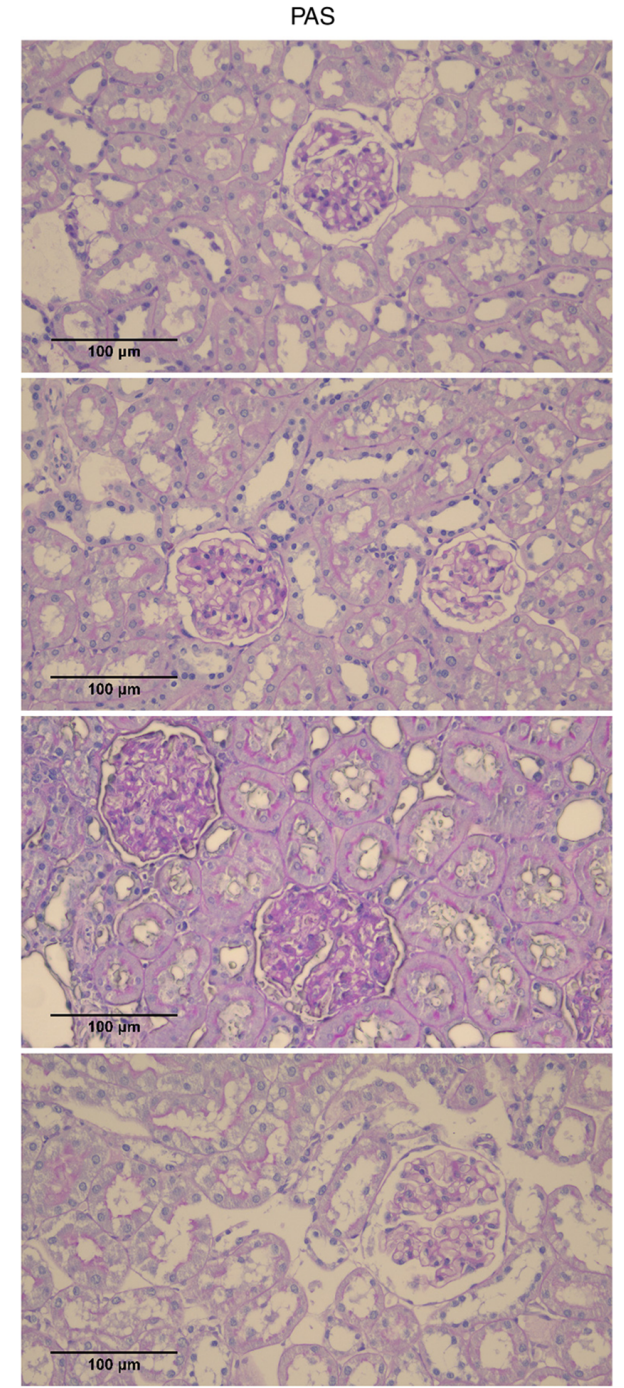

D

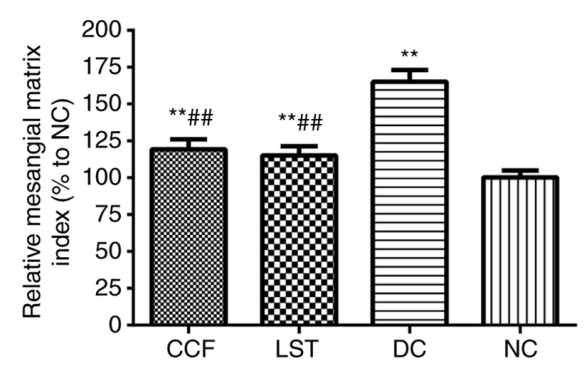

Figure 3. (A) Histopathological changes in the kidney were analysed by PAS staining (x400) and (B) H\&E staining (x200) in four groups of Sprague-Dawley rats $(n=7)$. (C) Relative glomerular volume and (D) relative mesangial matrix index were calculated in four groups ( $\mathrm{n}=7$ ). Data are expressed as the mean \pm standard deviation. ${ }^{* *} \mathrm{P}<0.01$ vs. the NC group; ${ }^{\#} \mathrm{P}<0.01$ vs. the DC group. Scale bar, $100 \mu \mathrm{m}$ for PAS staining images. Scale bar, $200 \mu \mathrm{m}$ for $\mathrm{H} \& \mathrm{E}$ staining imaging. Arrows indicate epithelial tubular cells with vacuolar degeneration. PAS, periodic acid-Schiff; CCF, compound centella formula; LST, losartan (4.5 mg/kg/day); DC, diabetic control; NC, normal control.

CCF reduces the UPCR in DKD rats. The results indicated that UPCR was significantly increased in the DC group compared with the NC group on day 112 (DC vs. NC; $\mathrm{P}<0.01$; Fig. 2A). The CCF group showed a significantly reduced UPCR compared with the DC group (CCF vs. DC; $\mathrm{P}<0.01)$. This effect was independent of blood sugar, since CCF did not notably reduce FBG (Fig. 2B). A similar response was exhibited in the LST group (LST vs. DC; $\mathrm{P}<0.01$; Fig. $2 \mathrm{~A}$ and $\mathrm{B})$. To investigate renal function in diabetic rats, Scr and BUN levels were measured. However, there was no significant difference between $\mathrm{NC}$ and $\mathrm{DC}$ (Fig. 2C and D).

CCF alleviates renal histological damage in DKD rats. Histopathological sections were stained with PAS (magnification, x400) and H\&E (magnification x200; Fig. 3A and B). The microscope observations indicated clear glomerular and renal tubular structures, with opened glomerular capillaries in the NC group rats. Conversely, the glomerular volume was significantly increased in the DC group rats compared with 
A

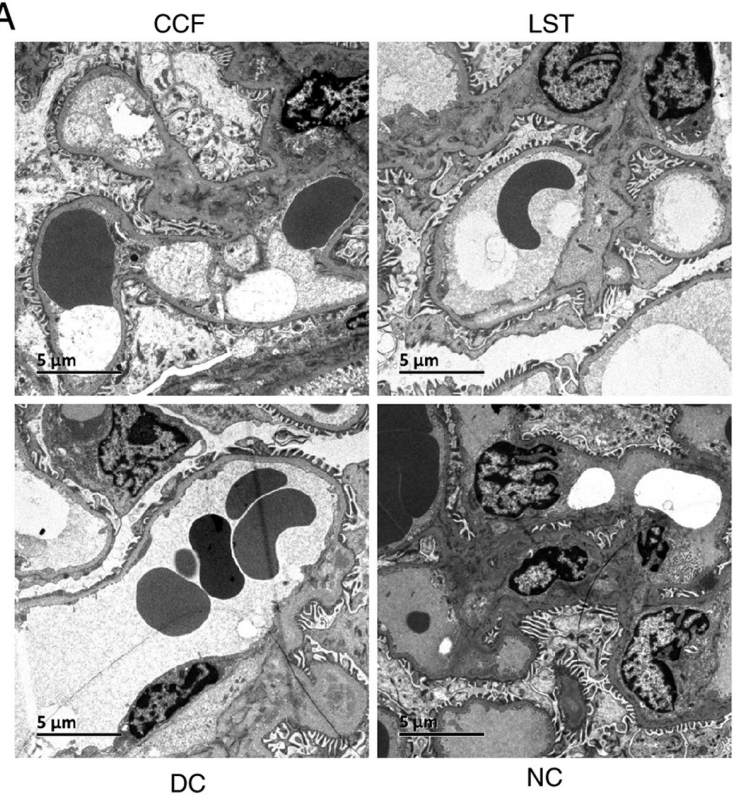

B

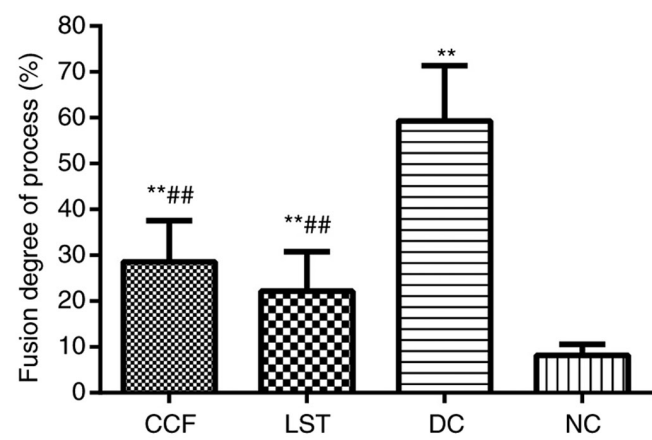

Figure 4. (A) Ultrastructural changes in the kidney were observed by transmission electron microscopy $(\mathrm{x} 4,000)$ in four groups of Sprague-Dawley rats $(n=7)(B)$ The fusion degree of the process was calculated in four groups $(n=7)$. Data are expressed as the mean \pm standard deviation. ${ }^{* *} \mathrm{P}<0.01$ vs. the $\mathrm{NC}$ group; ${ }^{\#} \mathrm{P}<0.01$ vs. the DC group. Scale bar, $5 \mu \mathrm{m}$. CCF, compound centella formula; LST, losartan $(4.5 \mathrm{mg} / \mathrm{kg} /$ day $)$; DC, diabetic control; NC, normal control.

the NC group rats. The basement membrane of the tubules and glomeruli was thickened with mesangial cell and stroma hyperplasia in the DC group. The relative glomerular volume and mesangial matrix index were increased in the DC group compared with the NC group (DC vs. NC; $\mathrm{P}<0.01$; Fig. $3 \mathrm{C}$ and D). Renal tubule vacuolization was also observed in the DC group. Some inflammatory cells could be found in the interstitial tissues or renal tubules. In the CCF group and LST group, these pathological changes were markedly improved (Fig. 3A and B).

Transmission electron microscopy showed that the foot process was complete and orderly in the NC group rats, without obvious glomerular basement membrane lesions. However, in the DC group, most of the foot processes were fused (Fig. 4A). The fusion degree of the process was increased in the DC group compared with the NC group (DC vs. NC; $\mathrm{P}<0.01$ ). After CCF or LST treatment, podocyte lesions were significantly improved (CCF vs. DC, P<0.01; LST vs. DC, P<0.01) (Fig. 4B).

CCF inhibits renal oxidative stress in DKD rats. As presented in Fig. 5A, the levels of SOD were significantly decreased in the
DC group compared with the $\mathrm{NC}$ group (DC vs. $\mathrm{NC} ; \mathrm{P}<0.01$ ), while MDA and NOX4 levels were increased (DC vs. NC; $\mathrm{P}<0.01$; Fig. 5B-D). CCF treatment partially restored the expression levels of SOD (CCF vs. DC; $\mathrm{P}<0.01)$ and decreased MDA (CCF vs. DC; $\mathrm{P}<0.01)$ and NOX4 (CCF vs. DC; $\mathrm{P}<0.01)$ to some extent. LST had a similar result (Fig. 5).

CCF attenuates renal inflammation in DKD rats.Quantification of TNF- $\alpha$ and NF- $\kappa$ B p65 suggested that the phosphorylation level of NF- $\kappa \mathrm{B}$ p65 was significantly upregulated in the DC group compared with the NC group (DC vs. NC; $\mathrm{P}<0.01$ ), while CCF treatment partially reduced the expression level to some extent (CCF vs. DC, $\mathrm{P}<0.01$; Fig. 6A). The level of TNF- $\alpha$ was also increased in the DC group compared with the $\mathrm{NC}$ group ( $\mathrm{DC}$ vs. $\mathrm{NC} ; \mathrm{P}<0.01$ ). $\mathrm{CCF}$ also significantly reduced the expression level of TNF- $\alpha(C C F$ vs. DC; $\mathrm{P}<0.01$; Fig. 6B). The expression trends of NF- $\mathrm{NB}$ p65 and TNF- $\alpha$ were similar in the CCF and LST groups.

$C C F$ increases the expression levels of SIRT1 $m R N A$ and protein in DKD rats. The mRNA levels of SIRT1 in rat renal tissues were significantly decreased in the DC group compared with the NC group (DC vs. NC; P<0.01; Fig. 7A). Treatment with either CCF or LST reversed this trend (CCF vs. DC; $\mathrm{P}<0.01$; LST vs. DC; $\mathrm{P}<0.01)$. The protein expression level of SIRT1 as measured by western blotting was decreased in the DC group compared with the NC group (DC vs. NC, $\mathrm{P}<0.01$; Fig. 7B). SIRT1 protein level was increased by CCF and LST treatment (CCF vs. DC; $\mathrm{P}<0.01$; LST vs. DC; $\mathrm{P}<0.01$; Fig. 7C).

Immunohistochemical staining of SIRT1 protein indicated that SIRT1 was mainly concentrated in the nucleus and cytoplasm of renal tubular epithelial cells, partially in the glomerulus, showing light or brown-yellow colour in the NC group (Fig. 8A). Slightly stained cells were observed in the DC group. The staining was increased in the CCF and LST groups compared with the DC group. The AOD of SIRT1 was the highest in the NC group and the lowest in the DC group (DC vs. NC; $\mathrm{P}<0.01$; Fig. 8B). The AOD in the CCF and LST groups was greater than that in the DC group (CCF vs. DC, $\mathrm{P}<0.01$; LST vs. $\mathrm{DC}, \mathrm{P}<0.01)$. There was no significant difference in the AOD of SIRT1 between CCF and LST.

\section{Discussion}

DKD is one of the most important long-term complications of diabetes and is a primary cause for dialysis (23-25). The classic pathological process of DKD includes glomerular hypertrophy, basement membrane and mesangial matrix thickening, typical nodular glomerular sclerosis and, as a final consequence, extensive glomerular sclerosis (26). Currently, there are two well-established types of rodent models for diabetes research. One type is the genetic spontaneous diabetes model, and the other is the experimentally induced diabetes models. The combination of high-fat diet with intraperitoneal STZ is widely used for inducing diabetes in rats (27). Previous research has indicated that male Wistar rats that received a high-fat diet for 4 weeks (28 days), followed by next day administration of STZ (35 mg/kg, via intraperitoneal injection), exhibited a fasting glucose level which was considered diabetic (28). According to the modelling method in previous literature, the rats in the DC, 
A

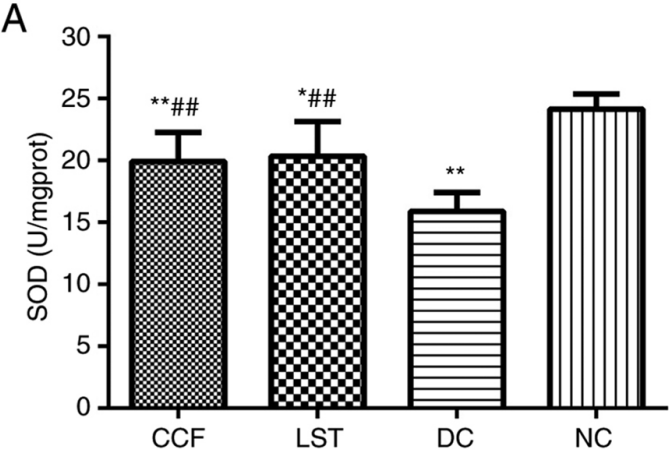

C

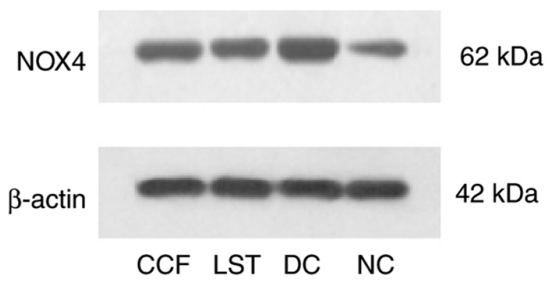

B

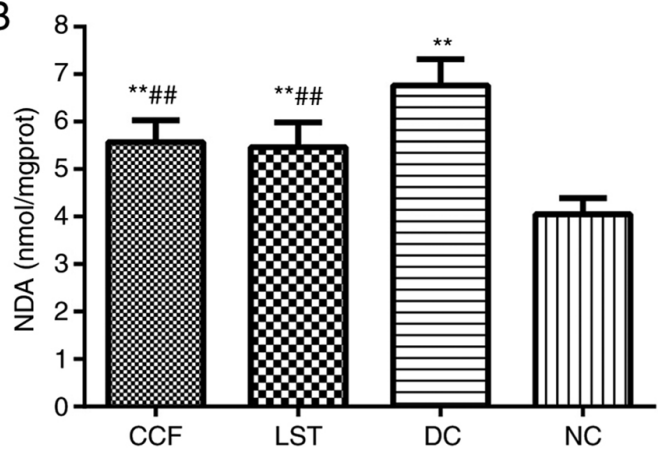

D

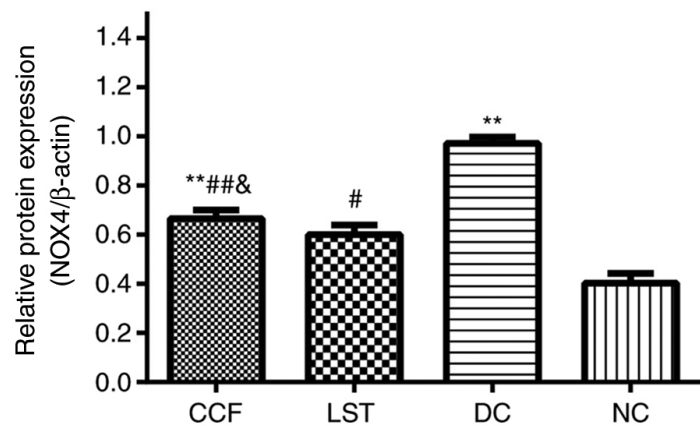

Figure 5. The levels of (A) SOD and (B) MDA in renal tissues were measured by an assay kit (n=7). (C and D) The protein expression of NOX4 was analysed by Western blotting in four groups of Sprague-Dawley rats $(n=7)$. Data are expressed as the mean \pm standard deviation. ${ }^{*} \mathrm{P}<0.05$ vs. the $\mathrm{NC}$ group; ${ }^{* *} \mathrm{P}<0.01 \mathrm{vs}$. the NC group; ${ }^{\text {P }}<0.05$ vs. the DC group; ${ }^{\#} \mathrm{P}<0.01$ vs. the DC group; ${ }^{\star} \mathrm{P}<0.05$ vs. the LST group. SOD, superoxide dismutase; MDA, malondialdehyde; NOX4, nicotinamide adenine dinucleotide phosphate oxidase 4; CCF, compound centella formula; LST, losartan (4.5 mg/kg/day); DC, diabetic control; $\mathrm{NC}$, normal control.

$\mathrm{CCF}$ and LST groups received a single intraperitoneal injection of $35 \mathrm{mg} / \mathrm{kg} \mathrm{STZ}$ the day following a 28-day high-fat diet in the present study. In previous studies, pathological changes were mainly observed 16 weeks after experimentally inducing diabetes in rats (presence of albuminuria, kidney histological changes including glomerulomegaly, inflammatory infiltration and tubulointerstitial fibrosis) $(29,30)$. Therefore, in the present study, all rats were sacrificed after 16 weeks, on the 112th day. In the present study, STZ-induced diabetic rats exhibited increased proteinuria, increased glomerulus, mesangial cell and stroma proliferation, renal tubule vacuolisation and foot process fusion. However, CCF reduced proteinuria and alleviated renal pathology. This renoprotection was independent of blood glucose control, as no significant decrease in blood sugar was observed.

Among numerous contributing factors, inflammatory and oxidative stress play crucial roles in the progression of DKD. Evidence indicates that low-grade inflammation usually exists in diabetic patients before the development of DKD (31). Inflammation has been observed in the serum and renal tissues of patients with $\mathrm{DKD}(32,33)$. NF- $\mathrm{kB}$ is a nuclear transcription factor that mainly regulates a large number of genes associated with inflammation and the immunological response (34). It is normally present in the cytoplasm in an inactive form. Activated NF- $\mathrm{KB}$ translocates into the nucleus and regulates the generation of proinflammatory cytokines, such as TNF- $\alpha$ (35). Although all of these cytokines are involved in the inflammatory response, TNF- $\alpha$ appears to be a critical mediator of the inflammatory cascade. A previous study has demonstrated that TNF- $\alpha$ reduced glomerular blood flow and glomerular filtration rate, and increased endothelin-1 production, inducing vasoconstriction, disrupting the glomerular filtration barrier which lead to proteinuria, and finally exerted direct apoptotic and cytotoxic effects on glomerular cells (36). One clinical trial indicated that TNF- $\alpha$ levels in urinary tissue were associated with the presence and severity of microalbuminuria in patients with type 2 diabetes mellitus (33). Consistent with clinical data, diabetic rats had a significantly higher increased level of urinary and renal interstitial concentrations of TNF- $\alpha$ before the increase in albuminuria (37). The present study demonstrated that the levels of the inflammatory factors NF- $\mathrm{kB}$ and TNF- $\alpha$ increased in the renal tissue of DKD rats, but significantly decreased with CCF treatment. This indicated that CCF may have a role in controlling the extent of the inflammatory response.

Oxidative stress plays another important role in the pathogenesis and progression of diabetes. Increased free radicals will interact with lipids, proteins and nucleic acids, leading to membrane integrity loss, structural or functional changes in proteins and gene mutation (38). MDA is one of the final products of polyunsaturated fatty acid peroxidation in cells, which affects ion exchange from the cell membranes, leading to cross-linking of the compounds located in the membrane as well as adverse consequences such as changing enzyme activity in parallel with ion permeability (39). MDA is an independent risk factor for DKD and reflects the level of lipid peroxidation and the severity of free radical attack on cells (40). NOX4 is a nicotinamide adenine dinucleotide phosphate oxidase subunit that is considered to be a main enzyme that contributes to increased oxidative stress in DKD. Previous studies have 

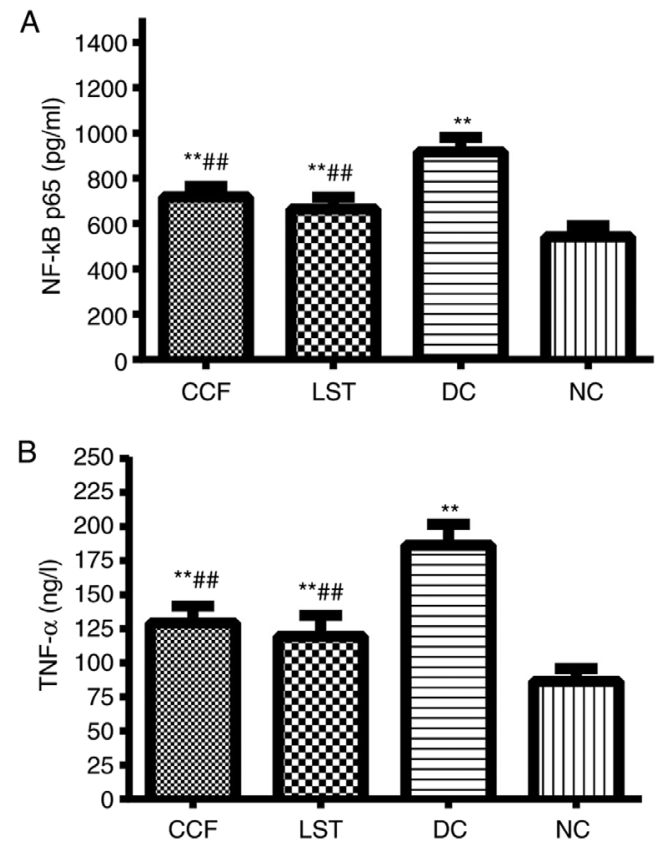

Figure 6. The levels of (A) NF- $\kappa$ B and (B) TNF- $\alpha$ were measured by ELISA in four groups of Sprague-Dawley rats $(n=7)$. Data are expressed as the mean \pm standard deviation., ${ }^{* *} \mathrm{P}<0.01$ vs. the $\mathrm{NC}$ group ${ }^{\# *} \mathrm{P}<0.01$ vs. the $\mathrm{DC}$ group. CCF, compound centella formula; LST, losartan $(4.5 \mathrm{mg} / \mathrm{kg} / \mathrm{day})$; DC, diabetic control; NC, normal control.

indicated that NOX4 expression is elevated in diabetic kidney injury, and podocyte-specific knockout of NOX4 attenuates DKD (41,42). However, humans possess enzymatic and nonenzymatic antioxidant defence systems to protect against the harmful effects of free radicals. For example, SOD can eradicate oxygen free radicals (43). Data generated from a previous study indicated that the level of SOD decreased in DKD animal models (44). In the present study, results revealed that the levels of NOX4 and MDA expression were increased, while the levels of SOD were decreased in DKD rats compared with control rats. After treatment with $\mathrm{CCF}$, these effects were reversed, and kidney condition was improved. Hence, it is hypothesised that the renoprotective effect of CCF may be mediated by antioxidants.

'OxInflammation' is a novel operative term that defines the deleterious crosstalk between inflammatory and redox systemic processes, leading to systemic or local damage in the long run (45). On the one hand, a feature of the inflammatory response is the generation of a pro-oxidative environment due to the production of pro-oxidant species. Increased production of TNF- $\alpha$ can stimulate the activation of nicotinamide adenine dinucleotide phosphate in mesangial cells and induce the production of ROS in endothelial cells. On the other hand, oxidative stress induces the release of inflammatory cytokines, such as TNF- $\alpha$ (46). The present results agree with these findings. The trend in inflammatory markers was consistent with the trend in oxidative stress markers and contrasted the trend in antioxidant markers. These results supported the existence of crosstalk between inflammatory and redox systemic processes.

SIRT1 is the most widely studied member of the SIRT family and is responsible for the deacetylation of proteins involved in the regulation of cell proliferation, cell
A

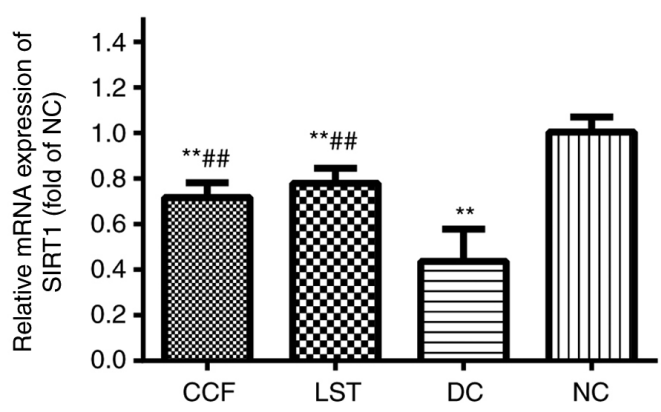

B

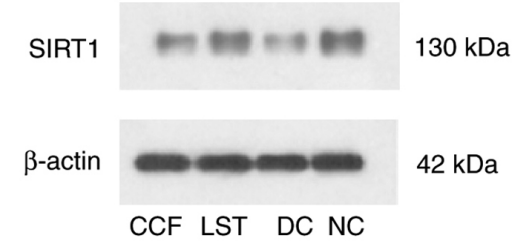

C

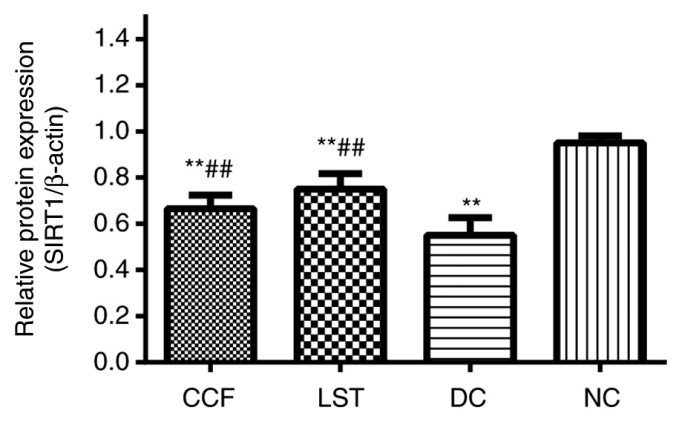

Figure 7. (A) The mRNA contents of SIRT1 in renal tissues were measured by quantitative PCR in four groups of Sprague-Dawley rats $(n=7)$. (B and C) The protein expression of SIRT1 in renal tissues was tested by western blotting in four groups of Sprague-Dawley rats $(n=7)$. Data are expressed as the mean \pm standard deviation. ${ }^{* *} \mathrm{P}<0.01$ vs. the $\mathrm{NC}$ group, ${ }^{\# \#} \mathrm{P}<0.01$ vs. the DC group. SIRT1, silent information regulator $1 ; \mathrm{CCF}$, compound centella formula; LST, losartan $(4.5 \mathrm{mg} / \mathrm{kg} / \mathrm{day})$; DC, diabetic control; NC, normal control.

differentiation, senescence, gene expression, mitochondrial biogenesis, fatty acid oxidation, apoptosis, autophagy and cellular metabolic balance $(10,47)$. The renoprotective effects of SIRT1 have been reported in various experimental models of renal disorders, including DKD $(12,13)$. A previous study has reported that decreased SIRT1 expression levels resulted in kidney dysfunction in diabetic rats, with increased proteinuria and decreased creatinine clearance. In contrast, the increase of SIRT1 expression levels mediated the hypertrophy of glomerular mesangial cells under diabetic conditions and alleviated renal damage (48). As a regulator of OxInflammation, previous studies have revealed some interesting outcomes regarding SIRT1. When SIRT1 levels decreased, oxidative stress and $\mathrm{NF}-\kappa \mathrm{B}$ levels increased in STZ-induced diabetic rats $(49,50)$. The activation of SIRT1 leads to NF- $\kappa \mathrm{B}$ deacetylation, thus reducing the release of inflammatory factors and decreasing the severity of diabetic nephropathy (51). Interestingly, a similar phenomenon was observed in the present study, in which renal SIRT1 levels decreased in the DC group and eventually increased with $\mathrm{CCF}$ treatment. Furthermore, it was also observed that CCF inhibited diabetes-induced oxidative damage and inflammatory factors and increased the expression level of SIRT1. 

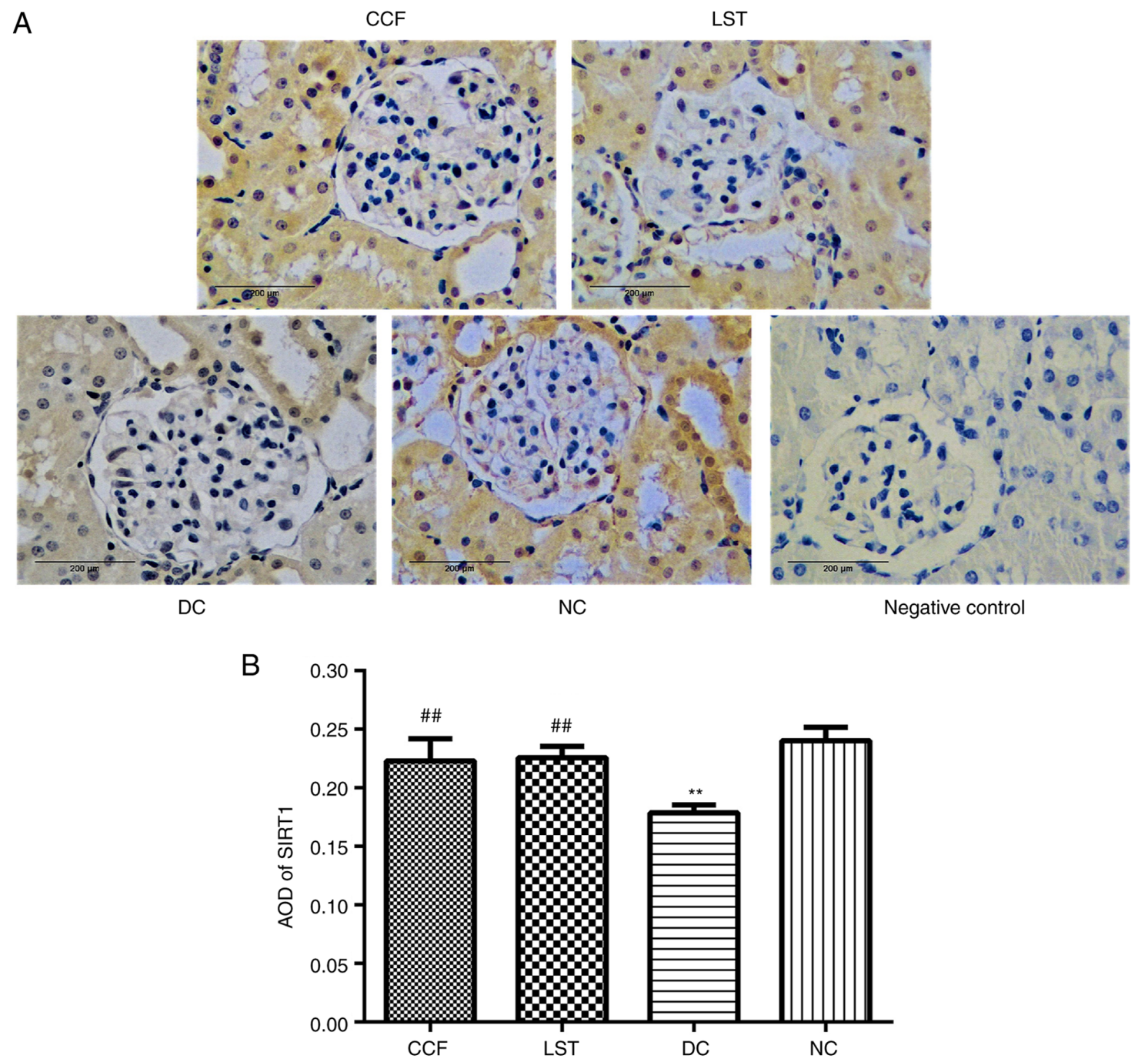

Figure 8. Protein expression and localisation of SIRT1 in renal tissues were observed by immunohistochemical staining in four groups of Sprague-Dawley rats $(n=7)$. (A) Positive staining is shown in yellow. (B) The AOD results were calculated in four groups $(n=7)$. Data are expressed as the mean \pm standard deviation. " P $<0.01$ vs. the NC group; ${ }^{\# \#} \mathrm{P}<0.01$ vs. the DC group. Scale bar, $200 \mu \mathrm{m}$. SIRT1, silent information regulator 1; CCF, compound centella formula; LST, losartan (4.5 mg/kg/day); DC, diabetic control; NC, normal control.

In conclusion, based on the results of the present study and data available in the literature, it is suggested that OxInflammation is involved in the progression of DKD as a consequence of the crosstalk between inflammatory and oxidative stress mediators. Moreover, SIRT1 may play an important role in regulating OxInflammation. CCF efficiently protected the kidney from diabetes, and the mechanism could be linked to the inhibition of OxInflammation and the upregulation of SIRT1. However, the necessary constituents of CCF require further study, and in vitro experiments will be performed to investigate the exact target of CCF.

\section{Acknowledgements}

Not applicable.

\section{Funding}

This research was financially supported by the Zhejiang Provincial Natural Science Foundation of China (grant no. LQ19H290005), the National Natural Science Foundation of China under (grant no. 81973760) and the Science and Technology Program for Health and Family Planning of Hangzhou (grant no. 2017A58).

\section{Availability of data and materials}

The datasets used and/or analysed during the current study are available from the corresponding author on reasonable request.

\section{Authors' contributions}

HYC, QYJ and QZ contributed to the conception and design of the study, and confirmed the authenticity of all raw data. XHL performed the histological analysis of the kidney. QZ was a major contributor in writing the manuscript. All authors read and approved the final manuscript.

\section{Ethics approval and consent to participate}

All procedures performed in studies involving animals were in accordance with the ethics standards of Zhejiang Chinese 
Medical University. This article does not contain any studies with human participants performed by any of the authors.

\section{Patient consent for publication}

Not applicable.

\section{Competing interests}

The authors declare that they have no competing interests.

\section{References}

1. König A, Schwarzinger B, Stadlbauer V, Lanzerstorfer P, Iken M, Schwarzinger C, Kolb P, Schwarzinger S, Mörwald K Brunner S, et al: Guava (Psidium guajava) fruit extract prepared by supercritical $\mathrm{CO}_{2}$ extraction inhibits intestinal glucose resorption in a double-blind, randomized clinical study. Nutrients 11: 1512,2019

2. Bakris GL, Hahr A, Khardori R, Koya D, Molitch M, Prischl FC, Schernthaner G and Thajudeen B: Managing diabetic nephropathies in clinical practice. Overview of diabetic nephropathy. 10.1007/978-3-319-08873-0: 1-21, 2017.

3. Varga ZV, Giricz Z, Liaudet L, Haskó G, Ferdinandy P and Pacher P: Interplay of oxidative, nitrosative/nitrative stress, inflammation, cell death and autophagy in diabetic cardiomyopathy. Biochim Biophys Acta 1852: 232-242, 2014

4. Flyvbjerg A: The role of the complement system in diabetic nephropathy. Nat Rev Nephrol 13: 311-318, 2017.

5. Choi JS, Kim J, Park J, Pyo S, Hong YK, Ku S and Kim MR: Blood glycemia-modulating effects of melanian snail protein hydrolysates in mice with type II diabetes. Int J Mol Med 39: 1437-1451, 2017.

6. Mizuno Y, Yamamotoya T, Nakatsu Y, Ueda K, Matsunaga Y, Inoue MK, Sakoda H, Fujishiro M, Ono H, Kikuchi T, et al Xanthine oxidase inhibitor febuxostat exerts an anti-inflammatory action and protects against diabetic nephropathy development in KK-Ay obese diabetic mice. Int J Mol Sci 20: 4680, 2019.

7. Rajesh M, Mukhopadhyay P, Bátkai S, Patel V, Saito K, Matsumoto S, Kashiwaya Y, Horváth B, Mukhopadhyay B, Becker L, et al: Cannabidiol attenuates cardiac dysfunction, oxidative stress, fibrosis, and inflammatory and cell death signaling pathways in diabetic cardiomyopathy. J Am Coll Cardiol 56: 2115-2125, 2010

8. Duecker R, Baer P, Eickmeier O, Strecker M, Kurz J, Schaible A, Henrich D, Zielen S and Schubert R: Oxidative stress-driven pulmonary inflammation and fibrosis in a mouse model of human ataxia-telangiectasia. Redox Biol 14: 645-655, 2018.

9. Valacchi G, Virgili F, Cervellati C and Pecorelli A: OxInflammation: From subclinical condition to pathological biomarker. Front Physiol 9: 858, 2018.

10. Bonkowski M and Sinclair D: Slowing ageing by design: The rise of NAD ${ }^{+}$and sirtuin-activating compounds. Nat Rev Mol Cell Biol 17: 679-690, 2016.

11. Guo R, Liu W, Liu B, Zhang B, Li W and Xu Y: SIRT1 suppresses cardiomyocyte apoptosis in diabetic cardiomyopathy: An insight into endoplasmic reticulum stress response mechanism. Int J Cardiol 191: 36-45, 2015.

12. Hao CM and Haase V: Sirtuins and their relevance to the kidney. J Am Soc Nephrol 21: 1620-1627, 2010.

13. Wakino S, Hasegawa $\mathrm{K}$ and Itoh $\mathrm{H}$ : Sirtuin and metabolic kidney disease. Kidney Int 88: 691-698, 2015.

14. Zhu Q, Zeng J, Li J, Chen X, Miao J, Jin Q and Chen H: Effects of compound centella on oxidative stress and Keap1-Nrf2-ARE pathway expression in diabetic kidney disease rats. Evid Based Complement Alternat Med 2020: 9817932, 2020.

15. Mengjie Z, Ziyang B, Liqiang Y, Danfeng G, Lin W and Jiao Z: Clinical Study on Modified Compound Jixuecao Tang for Chronic Glomerulonephritis and Chronic Kidney Disease at the Third Stage. Journal of New Chinese Medicine: 2019.

16. Spiers DE and Candas V: Relationship of skin surface area to body mass in the immature rat: A reexamination. J Appl Physiol Respir Environ Exerc Physiol 56: 240-243, 1984.

17. Ding Y, Zhang R, Zhang K, Lv X, Chen Y, Li A, Wang L, Zhang X and Xia Q: Nischarin is differentially expressed in rat brain and regulates neuronal migration. PloS One 8: e54563, 2013.
18. Zhu X, Chen Y, Chen Q, Yang H and Xie X: Astaxanthin promotes Nrf2/ARE signaling to alleviate renal fibronectin and collagen IV accumulation in diabetic rats. J Diabetes Res 2018: 6730315,2018

19. Artacho-Perula E, Roldan-Villalobos R, Salcedo-Leal I and Vaamonde-Lemos R: Stereological estimates of volume-weighted mean glomerular volume in streptozotocin-diabetic rats. Lab Invest 68: 56-61, 1993.

20. Liu HF, Guo LQ, Huang YY, Chen K, Tao JL, Li SM and Chen XW: Thiazolidinedione attenuate proteinuria and glomerulosclerosis in Adriamycin-induced nephropathy rats via slit diaphragm protection. Nephrology (Carlton) 15: 75-83, 2010.

21. Loeffler I and Wolf G: Pathophysiologie der diabetischen Nephropathie. Der Nephrologe 12: 391-399, 2017.

22. Karolina L, Hannes O, Risul A, Arvind P, Regina G, Taylor RF, Moosa M, Ann C, Karolina K and Larsson TE: Arterial klotho expression and FGF23 effects on vascular calcification and function. Plos One 8: e60658, 2013.

23. Martynyuk L, Martynyuk L, Ruzhytska O and Martynyuk O: Effect of the herbal combination canephron $\mathrm{N}$ on diabetic nephropathy in patients with diabetes mellitus: Results of a comparative cohort study. J Altern Complement Med 20: 472-478, 2014.

24. Tavafi M: Diabetic nephropathy and antioxidants. J Nephropathology 2: 20-27, 2013.

25. Gosmanov AR, Wall BM and Gosmanova EO: Diagnosis and treatment of diabetic kidney disease. Am J Med Sci 347: 406-413, 2014.

26. Sego S: Pathophysiology of diabetic nephropathy. Nephrol Nurs J 34: 631-633, 2008 .

27. Magalhães DA, Kume WT, Correia FS, Queiroz TS, Allebrandt Neto EW, Santos MP, Kawashita NH and França SA: High-fat diet and streptozotocin in the induction of type 2 diabetes mellitus: A new proposal. An Acad Bras Ciênc 91: e20180314, 2019.

28. Guex CG, Reginato FZ, de Jesus PR, Brondani JC, Lopes GH and Bauermann LF: Antidiabetic effects of Olea europaea L. leaves in diabetic rats induced by high-fat diet and low-dose streptozotocin. J Ethnopharmacol 235: 1-7, 2019.

29. Ahad A, Ganai AA, Mujeeb M and Siddiqui WA: Ellagic acid, an NF- $\kappa \mathrm{B}$ inhibitor, ameliorates renal function in experimental diabetic nephropathy. Chemico-Biological Interactions 219: 64-75, 2014.

30. Ahad A, Ganai AA, Mujeeb M and Siddiqui WA: Chrysin, an anti-inflammatory molecule, abrogates renal dysfunction in type 2 diabetic rats. Toxicol Appl Pharmacol 279: 1-7, 2014.

31. Serdar M, Sertoglu E, Uyanik M, Tapan S, Bilgi C and Kurt I: Comparison of 8-hydroxy-2'-deoxyguanosine (8-OHdG) levels using mass spectrometer and urine albumin creatinine ratio as a predictor of development of diabetic nephropathy. Free Radic Res 46: 1291-1295, 2012.

32. Yang M, Jun L, Zhou X, Ding H, Xu J, Yang B, Sun B, Xiao D, Yu J and Gong Q: Correlation analysis between serum vitamin D levels and lower extremity macrovascular complications in individuals with type 2 diabetes mellitus. J Diabetes Res 2019: 4251829, 2019.

33. Lampropoulou IT, Stangou M, Papagianni A, Didangelos T, Iliadis F and Efstratiadis G: TNF- $\alpha$ and microalbuminuria in patients with type 2 diabetes mellitus. J Diabetes Res 2014: $394206,2014$.

34. Malikova J, Zdarilova A and Hlobilkova A: Effects of sanguinarine and chelerythrine on the cell cycle and apoptosis. Biomed Pap Med Fac Univ Palacky Olomouc Czech Repub 150: 5-12, 2006.

35. Liu T, Zhang L, Joo D and Sun SC: NF- $\kappa \mathrm{B}$ signaling in inflammation. Signal Transduct Target Ther 2: 17023, 2017.

36. Xu XY and Ye SW: Efficacy assessment of treating post-stroke shoulder-hand syndrome patients of yin deficiency yang hyperactivity with blood stasis stagnation collaterals syndrome by yishen tongluo decoction. Zhongguo Zhong Xi Yi Jie He Za Zhi 34: 1069-1073, 2014 (Article in Chinese).

37. Kalantarinia K, Awad AS and Siragy HM: Urinary and renal interstitial concentrations of TNF- $\alpha$ increase prior to the rise in albuminuria in diabetic rats. Kidney Int 64: 1208-1213, 2003.

38. Takebayashi K, Matsumoto S, Aso Y and Inukai T: Aldosterone blockade attenuates urinary monocyte chemoattractant protein-1 and oxidative stress in patients with type 2 diabetes complicated by diabetic nephropathy. J Clin Endocrinol Metab 91: 2214-2217, 2006. 
39. Kowalczuk K and Stryjecka-Zimmer M: The influence of oxidative stress on the level of malondialdehyde (MDA) in different areas of the rabbit brain. Ann Univ Mariae Curie Sklodowska Med 57: 160-164, 2002.

40. Kaefer M, De Carvalho JA, Piva SJ, da Silva DB, Becker AM, Sangoi MB, Almeida TC, Hermes CL, Coelho AC, Tonello R, et al: Plasma malondialdehyde levels and risk factors for the development of chronic complications in type 2 diabetic patients on insulin therapy. Clin Lab 58: 973-978, 2012.

41. Li X, Cai W, Lee K, Liu B, Deng Y, Chen Y, Zhang X, He J and Zhong Y: Puerarin attenuates diabetic kidney injury through the suppression of NOX4 expression in podocytes. Sci Rep 7: 14603, 2017.

42. Ribaldo PD, Souza DS, Biswas SK, Block K, Faria J, Lopes de Faria JM and Lopes de Faria JB: Green tea (Camellia sinensis) attenuates nephropathy by downregulating Nox4 NADPH oxidase in diabetic spontaneously hypertensive rats. J Nutr 139: 96-100, 2009.

43. Peng Q, Liu F and Liang X: Superoxide dismutase and plant resistance to the environmental stress. Heilongjiang Agricultural Science 1: 31-34, 2002.

44. Samarghandian S, Borji A, Delkhosh M and Samini F: Safranal treatment improves hyperglycemia, hyperlipidemia and oxidative stress in streptozotocin-induced diabetic rats. J Pharm Pharm Sci 16: 352-362, 2013.

45. Tisato V, Gallo S, Melloni E, Celeghini C, Passaro A, Zauli G, Secchiero P, Bergamini C, Trentini A, Bonaccorsi G, et al: TRAIL and ceruloplasmin inverse correlation as a representative crosstalk between inflammation and oxidative stress. Mediators Inflamm 2018: 9629537, 2018.
46. Elmarakby AA and Sullivan JC: Relationship between oxidative stress and inflammatory cytokines in diabetic nephropathy. Cardiovasc Ther 30: 49-59, 2012

47. Webster BR, Lu Z, Sack MN and Scott I: The role of sirtuins in modulating redox stressors. Free Radical Biol Med 52: 281-290, 2011.

48. Yang X, Zhang B, Lu X, Yan M, Wen Y, Zhao T and Li P: Effects of Tangshen Formula on urinary and plasma liver-type fatty acid binding protein levels in patients with type 2 diabetic kidney disease: Post-hoc findings from a multi-center, randomized, double-blind, placebo-controlled trial investigating the efficacy and safety of Tangshen Formula in patients with type 2 diabetic kidney disease. BMC Complement Altern Med 16: 246, 2016

49. Iskender H, Dokumacioglu E, Sen TM, Ince I, Kanbay Y and Saral S: The effect of hesperidin and quercetin on oxidative stress, NF- $\mathrm{BB}$ and SIRT1 levels in a STZ-induced experimental diabetes model. Biomed Pharmacother 90: 500-508, 2017.

50. Huang K, Gao X and Wei W: The crosstalk between Sirt1 and Keap1/Nrf2/ARE anti-oxidative pathway forms a positive feedback loop to inhibit FN and TGF- $\beta 1$ expressions in rat glomerular mesangial cells. Exp Cell Res 361: 63-72, 2017.

51. Du YG, Zhang KN, Gao ZL, Dai F, Wu XX and Chai KF: Tangshen formula improves inflammation in renal tissue of diabetic nephropathy through SIRT1/NF- $\mathrm{kB}$ pathway. Exp Ther Med 15: 2156-2164, 2018.

(c) (7) $(9)$ This work is licensed under a Creative Commons

cc) Attribution-NonCommercial-NoDerivatives 4.0 International (CC BY-NC-ND 4.0) License. 